\title{
Understanding lupus nephritis: diagnosis, management, and treatment options
}

This article was published in the following Dove Press journal:

International Journal of Women's Health

21 May 2012

Number of times this article has been viewed

\section{Chi Chiu Mok}

Department of Medicine, Tuen Mun Hospital and Center for Assessment and Treatment of Rheumatic Diseases, Pok Oi Hospital, Hong Kong, China
Correspondence: Chi Chiu Mok

Chief of Rheumatology, Department of Medicine and Geriatrics, Tuen Mun Hospital, Tsing Chung Koon Road, New Territories, Hong Kong, China

Tel +l 85224685389

$\mathrm{Fax}+\mathrm{I} 85224569100$

Email ccmok2005@yahoo.com

\begin{abstract}
Systemic lupus erythematosus (SLE) predominantly affects women in their reproductive years. Renal disease (glomerulonephritis) is one of the most frequent and serious manifestations of SLE. Of the various histological types of lupus glomerulonephritis, diffuse proliferative nephritis carries the worst prognosis. Combined with high-dose prednisone, mycophenolate mofetil (MMF) has emerged as a first-line immunosuppressive treatment, although data regarding the efficacy of MMF on the long-term preservation of renal function are forthcoming. Cyclophosphamide is reserved for more severe forms of lupus nephritis, such as crescentic glomerulonephritis with rapidly deteriorating renal function, patients with significant renal function impairment at presentation, and refractory renal disease. Evidence for the calcineurin inhibitors in the treatment of lupus nephritis is weaker, and it concerns patients who are intolerant or recalcitrant to other agents. While further controlled trials are mandatory, B cell modulation therapies, such as rituximab, belimumab and epratuzumab are confined to refractory disease. Non-immunosuppressive measures, such as angiotensin-converting enzyme inhibitors, vigorous blood pressure control, prevention and treatment of hyperlipidemia and osteoporosis, are equally important.
\end{abstract}

Keywords: lupus, nephritis, nephropathy, glomerulonephritis, treatment, therapy, women

\section{Introduction}

Systemic lupus erythematosus (SLE) is a multi-systemic autoimmune disease of unknown etiology. In all series of SLE worldwide, women constitute more than $90 \%$ of all patients. This female preponderance becomes less pronounced before puberty and after menopause, which suggests that estrogen metabolism and its link with the immune system may play roles in the pathogenesis of the disease. ${ }^{1}$ An alternative hypothesis for the sexual dimorphism of SLE is the skewed inactivation or monosomy of the $\mathrm{X}$ chromosome, which contains immune-related genes. ${ }^{2}$ Although the exact pathoetiologic mechanisms have yet to be elucidated, it is believed that the onset of SLE is triggered by ill-defined environmental factors in genetically susceptible individuals.

Renal disease is one of the most common and most serious manifestations of SLE. Renal involvement in SLE adversely affects its ultimate prognosis in terms of patient survival and renal survival (survival without the need for renal replacement therapy) rates, as well as quality of life, including work disability. ${ }^{3}$ The glomerulus is the most common site of kidney involvement by lupus. However, the renal interstitium and tubules, as well as the vasculature, may also be affected. ${ }^{4}$ Early recognition of renal disease and close monitoring for progress after treatment is an essential part of management. Conventional serological markers and clinical renal parameters for active 
lupus nephritis are not sensitive or specific enough, and novel biomarkers for early detection of renal disease and prediction of renal prognosis are under current evaluation. ${ }^{5}$

In this review, the prevalence, presentation, and treatment of lupus renal disease is summarized based on information from recent clinical observation and controlled trials.

\section{How common is renal disease in SLE?}

Lupus renal disease appears to be more prevalent in certain ethnic groups. ${ }^{6,7}$ A comparative study of SLE in three ethnic groups reported that renal disease, which is defined by American College of Rheumatology (ACR) criteria as persistent daily proteinuria of $>500 \mathrm{mg}$ in the presence of cellular casts or biopsy evidence of lupus nephritis, occurred in $45 \%$ of African Americans, $42 \%$ of Chinese, and $30 \%$ of Caucasian patients, respectively. ${ }^{6}$ Another multi-ethnic US cohort of SLE patients reported that renal disease occurred in $51 \%$ of Africans and $43 \%$ of Hispanics but in only $14 \%$ of Caucasians. ${ }^{8}$ In a prospective study of 216 Chinese patients with new onset SLE, $31 \%$ patients had active renal disease as the initial presentation. ${ }^{9}$ The overall cumulative incidence of renal disease was $60 \%$ at 5 years post-SLE diagnosis. These studies illustrated that lupus renal involvement is more common in Africans, Hispanics, and Chinese than in Caucasians.

\section{Clinical presentation of lupus nephritis}

The presentation of renal disease in SLE is variable, ranging from no symptoms (detected by routine renal biopsy or "silent" lupus nephritis), trace proteinuria or active urinary sediments (microscopic hematuria, pyuria or cellular casts), to more serious proteinuria (nephrotic syndrome) and acute nephritic syndrome with rapid progression to acute renal failure. Occasionally, patients may present with chronic renal failure, isolated renal insufficiency, and hypertension as the initial manifestation.

The wide range of presentations of lupus nephritis does not necessarily correlate with the renal histological findings. A retrospective study of 21 SLE patients with low levels of proteinuria $(<1 \mathrm{~g} /$ day $)$ who underwent renal biopsy showed that proliferative lupus nephritis was present in $57 \%$ of patients. ${ }^{10}$ This emphasizes the importance of renal biopsy, especially for new onset renal disease with active lupus serology.

\section{The value of renal biopsy}

Renal biopsy is the gold standard of confirming the diagnosis and flare of lupus glomerulonephritis. The finding of positive staining for immunoglobulin $\mathrm{G}, \mathrm{A}$, and $\mathrm{M}$ with $\mathrm{C} 1 \mathrm{q}, \mathrm{C} 3$, and $\mathrm{C} 4$ constitutes the "full house" staining pattern for lupus nephritis. In addition, in guiding therapeutic decisions, renal biopsy provides information on the histological classes of lupus nephritis, in addition to the degree of inflammation and damage in the kidneys. Renal biopsy should be considered in SLE patients with new onset of proteinuria of more than $1 \mathrm{~g}$ /day with and without active urinary sediments, particularly in the presence of active lupus serology or impaired renal function. Some experts recommend renal biopsy at a lower threshold of proteinuria (eg, $\geq 500 \mathrm{mg} /$ day).

Patients with lupus nephritis that is refractory to treatment should be evaluated for other possible causes for the persistence of proteinuria or deterioration in renal function, such as the nephrotoxic side effects of medications (eg, the calcineurin inhibitors and nonsteroidal anti-inflammatory drugs), renal vein thrombosis, infections, overdiuresis, and poorly controlled hypertension. Treatment compliance should be checked. A repeat renal biopsy should be considered in patients with persistently active serological markers because it provides information on the following: (1) histological transformation of the classes of lupus nephritis; (2) the degree of residual activity in the kidneys; and (3) the extent of chronic irreversible changes and their progression since the initiation of immunosuppressive treatment. These data may help guide further treatment decisions.

\section{Prognosis of lupus nephritis}

Lupus nephritis carries significant morbidity and mortality. In the 1990s, the renal survival (survival without dialysis) rates of lupus nephritis ranged from $83 \%$ to $92 \%$ in 5 years and $74 \%$ to $84 \%$ in 10 years. ${ }^{3,11-13}$ The risks of end stage renal failure were particularly high in patients with diffuse proliferative glomerulonephritis, with figures ranging from $11 \%$ to $33 \%$ in 5 years. ${ }^{3,7,11,13-16}$ The prognosis of lupus nephritis depends on a large number of demographic, racial, genetic, histopathological, immunological, and time-dependent factors. ${ }^{17}$ Renal disease that fails to remit with immunosuppressive therapies is a major risk factor for subsequent deterioration of renal function and poor outcome. ${ }^{3,16,18}$ Other unfavorable prognostic factors for lupus nephritis include younger age, male sex, histological cellular crescents, fibrinoid necrosis, subendothelial deposits, glomerular scarring, tubular atrophy and interstitial fibrosis, impaired renal function at presentation, persistent hypertension, hypocomplementemia, low hematocrit, in addition to delay in treatment due to limited access to health care and poor compliance. ${ }^{17}$ 
Table I ISN/RPS histological classification of lupus nephritis ${ }^{21}$

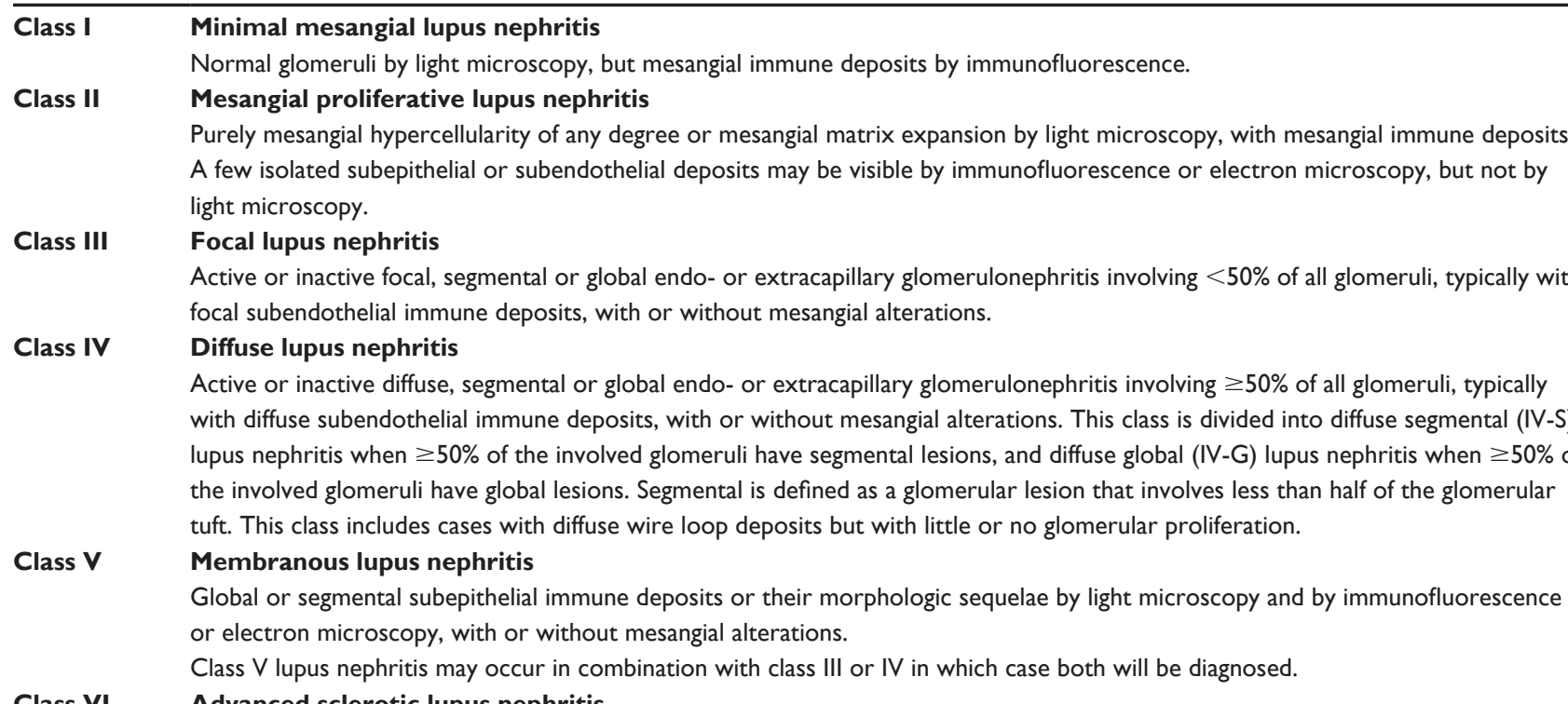

Class VI Advanced sclerotic lupus nephritis

$\geq 90 \%$ of glomeruli globally sclerosed without residual activity.

Abbreviation: ISN/RPS, International Society of Nephrology/Renal Pathology Society. Data drawn from Weening JJ, D’Agati VD, Schwartz MM, Seshan SV, Alpers CE, Appel $\mathrm{GB}$, et al. The classification of glomerulonephritis in systemic lupus erythematosus revisited. J Am Soc Nephrol. 2004;15:24I-250.21

\section{Contemporary treatment of lupus nephritis}

Therapy for lupus nephritis should aim at symptomatic control, preservation of renal function, reduction of renal flares, prevention of treatment-related complications, and ultimately, reduction in mortality. ${ }^{19}$ Immunosuppressive therapy for lupus nephritis is divided into two phases: the induction phase targets reducing inflammation and glomerular injury; and the maintenance phase aims to reduce long-term risks of renal flares and renal function decline.

Adjunctive therapies, such as vigorous control of blood pressure to $<120 / 80 \mathrm{mmHg}$, may retard the deterioration of renal function. The early use of renal protection agents, such as the angiotensin converting enzyme inhibitors (ACEIs) and the angiotensin II receptor antagonists, is mandatory. Hyperlipidemia should also be controlled to offer protection against accelerated vascular disease, particularly in the membranous type of lupus nephritis. Calcium and vitamin D should be adequately supplemented to reduce the risk of aggravation of disease activity related to vitamin D deficiency, ${ }^{20}$ and to protect against osteoporosis. Low-dose aspirin may be considered in patients with histological evidence of antiphospholipid syndrome nephropathy, although there is no published evidence to support this treatment. Anticoagulation may be considered in patients with persistent nephrotic ranges of proteinuria and the presence of antiphospholipid antibodies.

\section{Induction therapy of lupus nephritis}

The current histological classification of lupus nephritis is based on the recommendation of the International Society of Nephrology/Renal Pathology Society (ISN/RPS) in 2003 (Table 1). ${ }^{21}$ Milder forms of lupus nephritis (ISN/RPS Class I, II) are usually manageable with glucocorticoids. ${ }^{22}$ Azathioprine (AZA) can be added as a glucocorticoid-sparing agent and for the treatment of concomitant extra-renal manifestations. Mild class V disease can be treated with ACEIs. Proliferative lupus nephritis (class III and IV or mixed III/V and IV/V) and more serious class V (nephrotic range of proteinuria or deteriorating renal function) disease require more aggressive induction regimens that combine glucocorticoid and a non-glucocorticoid immunosuppressive agent. The standard induction therapy for severe lupus nephritis has been a combination of high-dose glucocorticoid and cyclophosphamide (CYC). A series of randomized controlled trials conducted by the National Institute of Health (NIH) demonstrated that prednisone combined with intravenous (IV) pulse CYC offered better long-term renal protection than prednisone monotherapy. ${ }^{23-25}$ However, the use of CYC is associated with a number of untoward side effects, which include infection, ovarian and bladder toxicities, leukopenia, increased risk of cervical intraepithelial neoplasia, and malignancy. Some of these toxicities are dose dependent, with higher risks related to higher cumulative doses ${ }^{26}{ }^{\mathrm{IV}}$ pulse CYC has gained popularity over daily oral $\mathrm{CYC}$ because it is associated with less toxicity in the bladder and the gonads. A recent analysis 
of a large cohort of patients with diffuse proliferative lupus nephritis showed a trend towards better efficacy of oral CYC than IV pulse $\mathrm{CYC}$ in preserving renal function after a mean follow-up of 8.8 years. ${ }^{18}$ In a multivariate model, a dose of CYC delivered cumulatively instead of the CYC route per se was independently associated with a complete renal response. However, ovarian toxicity leading to premature menopause was more frequent in users of oral CYC.

Although the optimal route and duration of CYC therapy in lupus nephritis remains uncertain, recent evidence supports the use of a shorter course and lower dose of CYC to minimize toxicities. ${ }^{27-29}$ Houssiau et al ${ }^{29}$ compared the efficacy and toxicity of two less intensive intravenous pulse CYC regimens for the initial treatment of lupus nephritis. Eighty-four patients (predominantly Caucasians) were randomized to receive either 8 intravenous pulses of CYC $\left(0.5 \mathrm{~g} / \mathrm{m}^{2}\right.$ to a maximum of $\left.1.5 \mathrm{~g}\right)$ or 6 biweekly low dose pulses of CYC (500 mg each). In both regimens, CYC was later substituted with AZA for long-term maintenance. Patients who participated in the study had milder renal disease compared to other lupus nephritis trials, as reflected by a lower proportion of patients having class IV disease, nephrotic syndrome, and renal function impairment. After 10 years, mortality, sustained doubling of serum creatinine, and end stage renal disease did not differ between the two groups. ${ }^{29}$ Thus, when there are no alternatives to CYC, a low-dose CYC regimen followed by AZA is a viable strategy for milder lupus nephritis. CYC is reserved for high-risk patients with proliferative lupus nephritis, such as those with impaired or rapidly deteriorating renal function, histological cellular crescents, or a combination of high activity and chronicity scores..$^{30}$ The course of CYC should be limited to less than 6 months, with subsequent replacement by another immunosuppressive agent to reduce toxicities. ${ }^{28}$

\section{Controlled trials for induction therapy of lupus nephritis}

Six randomized controlled trials for induction therapy of severe lupus nephritis were recently presented (Table 2). ${ }^{31-36}$ In the largest lupus nephritis controlled trial to-date, the Aspreva Lupus Management Study (ALMS), 370 patients with histologically ISN/RPS class III, IV, or V lupus nephritis ( $2 / 3$ class IV disease) were randomized to receive either monthly IV pulse CYC $\left(0.5-1.0 \mathrm{~g} / \mathrm{m}^{2}\right)$ or MMF (target $3 \mathrm{~g} /$ day) on top of high-dose prednisone $(60 \mathrm{mg} /$ day initially and then tapered). ${ }^{31}$ Asians and Hispanics comprised 33\% and $35 \%$ of the participants, respectively. Three hundred and six $(83 \%)$ patients completed the 24-week protocol.
Clinical response, defined by a decrease in urine protein/ creatinine ratio $(\mathrm{P} / \mathrm{Cr})$ to $<3$ in patients with baseline nephrotic range $\mathrm{P} / \mathrm{Cr} \geq 3$, or by $\geq 50 \%$ in patients with subnephrotic baseline $\mathrm{P} / \mathrm{Cr}(<3)$, and stabilization $( \pm 25 \%)$ or improvement in serum creatinine at 24 weeks as adjudicated by a blinded clinical endpoints committee, was not significantly different between the CYC (53\%) group and the MMF (56\%) group. Subgroup analyses revealed that MMF was associated with a significantly higher response rate than CYC (60\% vs 39\%; $P=0.03$ ) in the non-Caucaisan non-Asians, who were mainly Hispanics. The rates of adverse events and serious adverse events were similar in the two groups. Specifically, nausea, vomiting, and alopecia were numerically more frequent with CYC, whereas diarrhea was more commonly reported in MMF users. There were 9 and 5 deaths in the MMF group and the CYC group, respectively. Of the 9 deaths in the MMF users, 7 were Asians (mainly Chinese), suggesting that Asian patients tolerated high-dose prednisone and MMF ( $3 \mathrm{~g} /$ day) less well.

Grootscholten et al ${ }^{32}$ randomized 87 patients with proliferative lupus nephritis (class III and IV) to receive either oral prednisone combined with intravenous pulse CYC ( $750 \mathrm{mg} / \mathrm{m}^{2}$ monthly for 6 months and then quarterly for another 7 doses) or intravenous pulse methylprednisolone ( $1 \mathrm{~g}$ daily for 3 days for 9 pulses) together with AZA ( $2 \mathrm{mg} / \mathrm{kg} /$ day). At the end of the third year, both groups of patients received AZA for maintenance ( $2 \mathrm{mg} / \mathrm{kg} /$ day), with the dosage reduced to $1 \mathrm{mg} / \mathrm{kg} /$ day after 4 years. This study consisted of mainly Caucasian patients $(76 \%)$ who had serious renal disease (57\% hypertension, 53\% nephrotic syndrome, and $56 \%$ impaired creatinine clearance at presentation). After a median of 5 years, significantly more AZA-treated patients relapsed, and a numerically higher number of patients had renal function deterioration. Despite the use of a more intensive corticosteroid regimen in the AZA arm, the outcome was less satisfactory, indicating that AZA was inferior to CYC for more severe lupus nephritis.

Bao et al $^{33}$ randomized 40 patients with mixed proliferative and membranous lupus nephritis (ISN/RPS IV + V) ( $85 \%$ of patients with normal serum creatinine) to receive either IV pulse CYC $\left(0.5-1 \mathrm{~g} \cdot \mathrm{m}^{2}\right.$ monthly $)(\mathrm{N}=20)$ or low-dose combination of MMF (500 mg BD) and tacrolimus (Tac) ( $2 \mathrm{mg} \mathrm{BD})(\mathrm{N}=20)$, in addition to corticosteroids (daily pulse methylprednisolone $0.5 \mathrm{~g}$ for 3 days, followed by $0.6-0.8 \mathrm{mg} / \mathrm{kg} /$ day of oral prednisolone). At 6 months, the rate of complete response defined as daily proteinuria $<0.4 \mathrm{~g} /$ day with normal urinary sediments and stabilization of serum creatinine $(<15 \%$ increase) was 


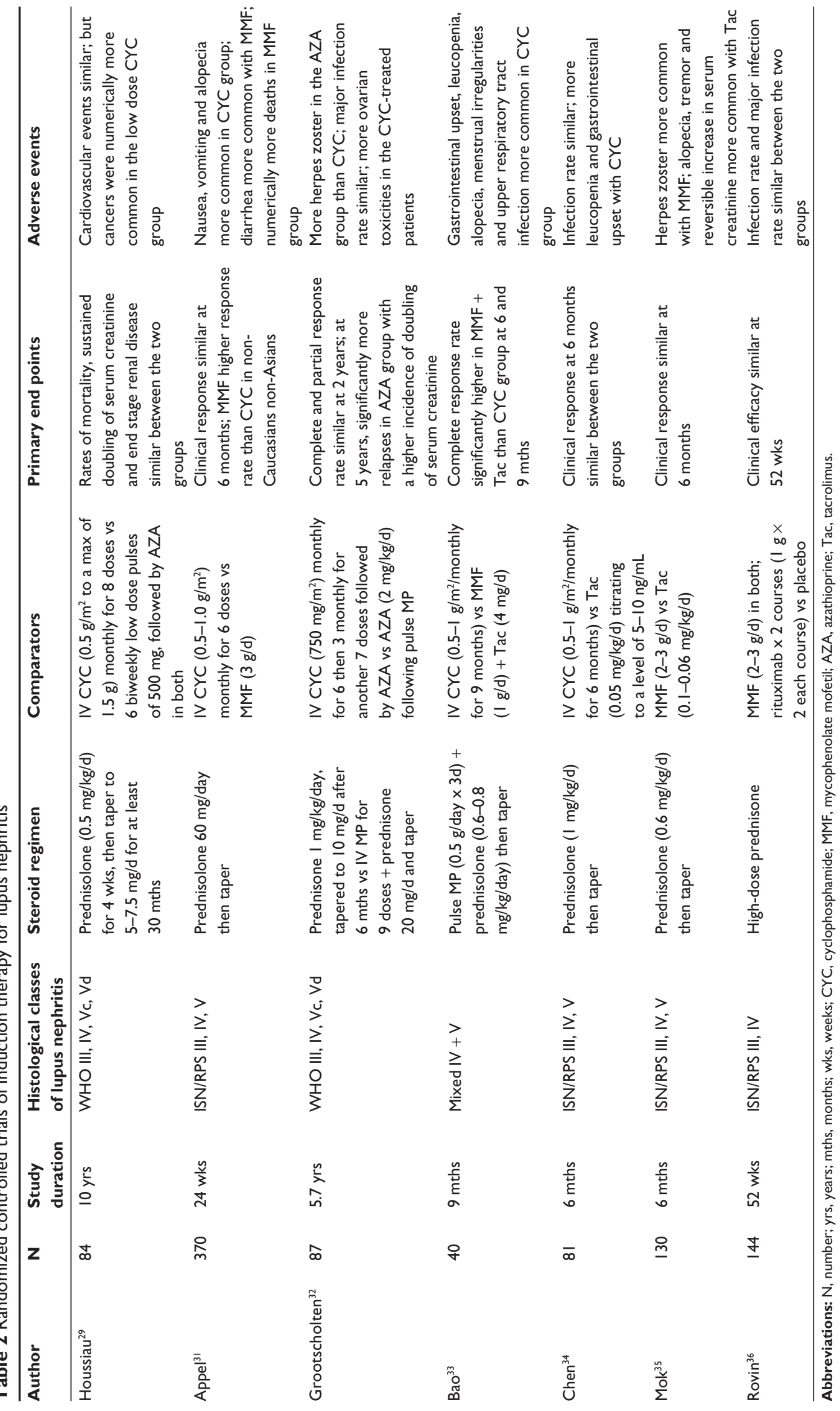


significantly higher in the MMF/Tac (50\%) group than the CYC (5\%) group. The corresponding rates at 9 months of treatment were $65 \%$ and $15 \%$, respectively. Leukopenia, gastrointestinal upset, upper respiratory tract infection, alopecia, and irregular menses were more common in the CYC group than in the MMF/Tac group of patients.

In a randomized controlled trial conducted recently by Chen et al, ${ }^{34} 81$ patients with class III, IV, or V lupus nephritis were randomized to receive IV pulse CYC $\left(0.5-1 \mathrm{~g} \cdot \mathrm{m}^{2}\right.$ monthly) $(\mathrm{N}=39)$ or Tac $(0.05 \mathrm{mg} / \mathrm{kg} /$ day titrating to a level of $>5 \mathrm{ng} / \mathrm{mL})(\mathrm{N}=42)$ in combination with highdose prednisolone $(1 \mathrm{mg} / \mathrm{kg} /$ day $)$. The study population consisted of moderate to high-risk patients ( $77 \%$ class IV disease and $11 \%$ impaired renal function at presentation). At 6 months, the rate of complete remission, which was defined as proteinuria $<0.3 \mathrm{~g} /$ day, stabilization of serum creatinine, and normalization of urinary sediments, was not significantly different in the CYC group and the Tac group of patients ( $38 \%$ vs $52 \%, P=0.2)$. Gastrointestinal upset and leucopenia were significantly more frequent in the CYC group, but the rate of infection was similar between the two arms. Transient increase in serum creatinine was reported in $8 \%$ of patients receiving Tac.

Our group conducted a controlled trial comparing the efficacy of MMF ( $2 \mathrm{~g}$ /day, titrating to $3 \mathrm{~g} /$ day if response is suboptimal at 3 months $)$ with Tac $(0.1 \mathrm{mg} / \mathrm{kg} /$ day in the first 2 months tapering to $0.06 \mathrm{mg} / \mathrm{kg} /$ day) in combination with high-dose prednisolone $(0.6 \mathrm{mg} / \mathrm{kg} / \mathrm{day}$ for 6 weeks and taper) for lupus nephritis. ${ }^{35}$ Our preliminary analysis of 130 patients showed that both complete and partial clinical response rates were not significantly different between the two treatment arms at 6 months. The rate of infection, in particular herpes zoster reactivation, was higher in MMF than in Tac-treated patients, whereas alopecia, tremor, and reversible increase in serum creatinine were more frequent in the Tac group of patients. Dose-related neurological and metabolic adverse effects of Tac and the possibility of early renal relapse upon completion of the induction phase and substitution of Tac must be carefully monitored.

The Lupus Nephritis Assessment with Rituximab (LUNAR) study is a phase III randomized, double-blind, placebo-controlled multicenter study to evaluate the efficacy and safety of rituximab in patients with active proliferative lupus nephritis. ${ }^{36}$ Patients with ISN/RPS Class III or IV lupus nephritis and urine protein to creatinine $(\mathrm{UP} / \mathrm{Cr})$ ratio $>1$ were randomized to receive rituximab $(1000 \mathrm{mg})(\mathrm{N}=72)$ or placebo $(\mathrm{N}=72)$ infusion on days 1, 15, 168 (week 24) and 182 (week 26), in addition to corticosteroid and MMF ( $>2 \mathrm{~g}$ /day).
Two-thirds of the patients had class IV nephritis. At week 52, no statistically significant differences in the primary and secondary endpoints were observed between the rituximab and placebo groups of patients, although there were numerically more responders in the rituximab group (57\% vs $46 \%$ in the placebo group), mainly among Africans and Hispanics. Serious adverse events and infection rates were similar between the two groups, but two deaths occurred in the rituximab-treated patients.

The information reviewed indicates that MMF should be used as the first-line treatment in combination with glucocorticoids for severe lupus nephritis because of the stronger evidence for it compared to other agents and the lower incidence of toxicities compared to conventional CYC. Although Tac has similar efficacy with either CYC or MMF, it has been tried in a smaller population of patients, and its long-term nephrotoxicity remains a concern. However, Tac is an option when patients are contraindicated for, intolerant to, or refractory to MMF. Tac is preferred to cyclosporin A because of the lower incidence of cosmetic side effects, particularly in young women. The initial results of the B cell depleting agents are disappointing. Although evidence does not support an additional benefit of rituximab, with MMF treatment for lupus nephritis, rituximab is still an option for recalcitrant lupus nephritis, as evidenced by a number of

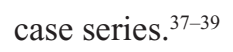

\section{Maintenance therapy of lupus nephritis}

Indirect evidence suggests that maintenance therapy is beneficial in severe lupus nephritis. In a long-term follow-up of 145 patients who participated in NIH lupus nephritis studies, renal flares occurred in $45 \%$ of patients when immunosuppression was completely stopped. ${ }^{40} \mathrm{~A}$ recent retrospective review of 32 patients with predominantly diffuse proliferative lupus nephritis described a relapse of lupus activity in 53\% of patients after immunosuppression was discontinued. ${ }^{41}$ In our experience with 212 patients with diffuse proliferative lupus nephritis, ${ }^{18}$ although maintenance treatment was given to $73 \%$ of patients, one-third of patients still had renal flares that might be serious. Maintenance therapy for $<3$ years was independently associated with an increased likelihood of having the composite outcome of doubling of serum creatinine, end stage renal failure, or death (hazard ratio 4.62 [1.35-15.8]; $P=0.02)$.

In Moroni's study, ${ }^{41}$ patients who experienced sustained remission of lupus nephritis had received a longer total median duration of immunosuppressive treatment than those 
who did not (median 57 months vs 30 months; $P<0.01$ ). This finding, coupled with the observation that maintenance treatment for $<3$ years after successful CYC induction was a predictor of poor renal outcome in proliferative lupus nephritis, ${ }^{18}$ suggests that maintenance immunosuppression should be continued for at least 3 years after a good clinical response is achieved.

Four randomized controlled trials on maintenance therapy of lupus nephritis are summarized in Table 3. Contreras et $\mathrm{al}^{42}$ randomized 59 patients with lupus nephritis (mainly African and Hispanic Americans; $78 \%$ had class IV disease) to receive one of the three treatment arms after induction with 4-7 pulses of intravenous CYC: (1) MMF (0.5-3 g/day); (2) quarterly pulse CYC; (3) AZA (1-3 mg/kg/day). Long-term observation showed that either MMF or AZA was superior to CYC in the prevention of the composite outcome of renal failure and death. MMF was more efficacious than pulse CYC in the prevention of renal flares. Moreover, maintenance treatment with CYC was associated with more side effects such as nausea, vomiting, and infection. Moroni et al ${ }^{43}$ studied 69 patients (mainly Caucasians) with lupus nephritis. After initial induction treatment with pulse methylprednisolone, prednisone, and oral CYC $(91.5 \pm 23.8 \mathrm{mg} /$ day for a median of 3 months), patients were randomized to receive either cyclosporin A (CSA) (Neoral; 4.0 to $2.5-3.0 \mathrm{mg} / \mathrm{kg} /$ day $)(\mathrm{N}=36)$ or AZA $(2 \mathrm{mg} / \mathrm{kg} /$ day $)$ $(\mathrm{N}=33)$ for maintenance. At 4 years of follow-up, flare occurred in $24 \%$ of AZA-treated patients and $19 \%$ of CSAtreated patients, respectively (no significant difference). Minor infections and leucopenia were more commonly reported with AZA treatment, whereas arthralgia and gastrointestinal symptoms were more common in CSA-treated patients.

In the MAINTAIN study conducted by Houssiau et al, ${ }^{44}$ 105 patients with class III, IV, Vc and Vd lupus nephritis were randomized to receive either MMF ( $2 \mathrm{~g}$ /day) $(\mathrm{N}=53)$ or AZA $(2 \mathrm{mg} / \mathrm{kg} /$ day $)(\mathrm{N}=52)$ after an initial induction regimen that consisted of IV pulse methylprednisolone, highdose prednisone and IV pulse CYC (500 mg twice weekly for 6 doses). Participants were mainly Caucasians, and 10\% of patients had impaired renal function at study entry. After a mean follow-up of 53 (15-65) months, 24 (23\%) patients withdrew from the study mainly because of pregnancy (in the MMF group) and adverse effects. Frequency of renal and extra-renal flares, doubling of serum creatinine, and incidence of infections occurred at similar frequency in the two arms. However, drug-related cytopenias were more common with AZA.

The maintenance phase of the ALMS study was recently published. ${ }^{45}$ Two hundred and twenty-seven patients

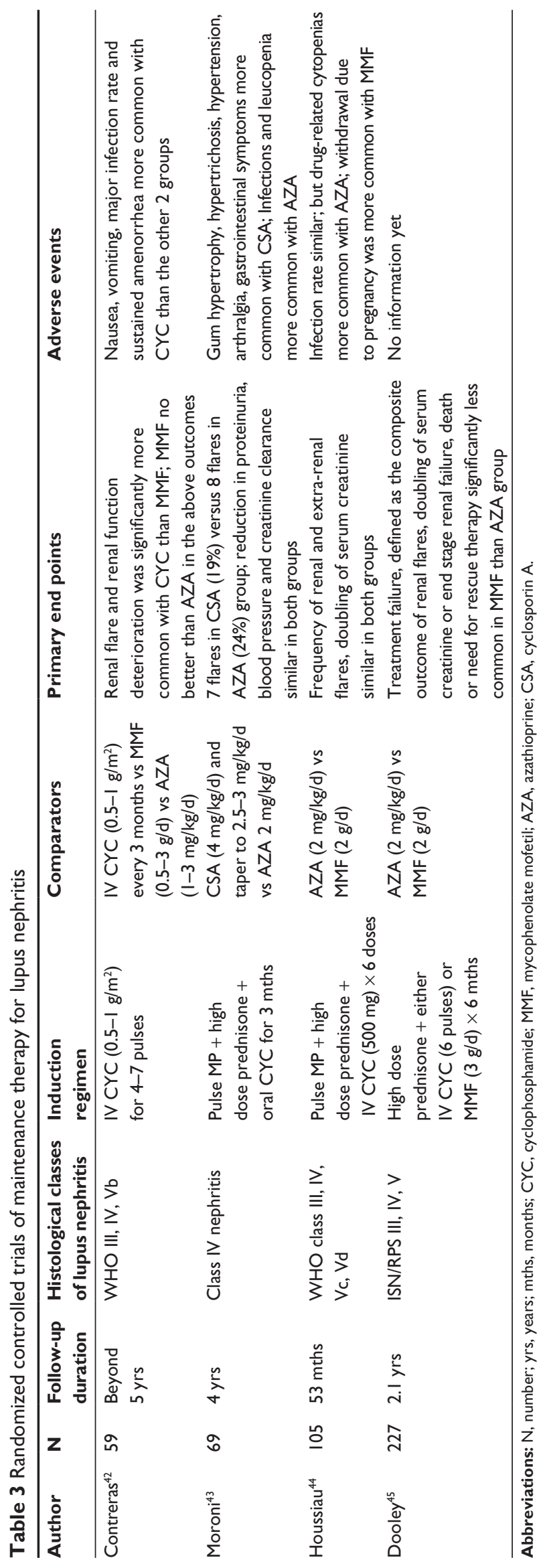


(44\% Whites, 10\% Blacks, and 33\% Asians) who had completed the induction phase of the ALMS (IV pulse CYC or MMF $3 \mathrm{~g}$ /day) were randomized to receive either MMF ( $2 \mathrm{~g} /$ day) $(\mathrm{N}=116)$ or AZA $(2 \mathrm{mg} / \mathrm{kg} /$ day $)(\mathrm{N}=111)$ for maintenance treatment. The mean daily doses received by the patients were $1.87 \pm 0.43 \mathrm{~g}$ and $120 \pm 48 \mathrm{mg}$, respectively, for MMF and AZA. After a mean follow-up of 2.1 years, the rate of treatment failure, defined as renal flare, doubling of serum creatinine, or end stage renal disease, needed for rescue therapy or death was significantly less common in MMF than in AZA-treated patients ( $16.4 \%$ vs $32.4 \% ; P=0.003)$. The results were similar regardless of induction by CYC or MMF, race, or geographical region.

In summary, it appears that MMF is the preferred agent for long-term maintenance therapy of lupus nephritis. However, its cost-effectiveness should be further evaluated. AZA and CSA are alternative options for patients who are intolerant to MMF or are planning for pregnancy. The longterm use of calcineurin inhibitors, such as Tac and CSA, must be cautious because of the increased risk of nephrotoxicity, hyperlipidemia, and atherosclerosis.

\section{Membranous lupus nephropathy}

Membranous lupus nephropathy (MLN) comprises only onefifth of all cases of histologically confirmed lupus nephritis. ${ }^{46}$ Reported rates of patient survival and end-stage renal disease in MLN vary considerably because of substantial heterogeneity among the published studies. The risk of progression of MLN to renal failure is generally reduced in the absence of proliferative lesions, but patients are nevertheless at risk for thromboembolic complications.

The optimal therapy for MLN remains elusive because of the paucity of clinical trials. Mixed membranous and proliferative lupus nephritis should be treated in the same way as pure proliferative lupus nephritis. If MLN is not accompanied by proliferative lesions but is associated with clinically relevant proteinuria, renal insufficiency, or failure to respond to supportive therapies, immunosuppressive treatment is indicated. In addition, cardiovascular protection and blockade of the renin-angiotensin system should be instituted early in all patients.

Austin et $\mathrm{a}^{47}$ randomized 42 patients $(71 \%$ Blacks or Hispanics) with MLN to receive one of the following regimens: (1) alternate day prednisone $(1 \mathrm{mg} / \mathrm{kg} /$ day for 8 weeks and taper to $0.25 \mathrm{mg} / \mathrm{kg} /$ day throughout); (2) similar prednisone regimen plus IV pulse CYC $\left(0.5-1.0 \mathrm{~g} / \mathrm{m}^{2}\right.$ every two months); or (3) similar prednisone regimen plus CSA $(5 \mathrm{mg} / \mathrm{kg} /$ day). At 12 months, the cumulative probability of complete $(<0.3 \mathrm{~g} /$ day proteinuria) or partial $(<2.0 \mathrm{~g} /$ day proteinuria or improvement by $50 \%$ from baseline) remission was highest with CSA (83\%), followed by IV pulse CYC $(60 \%)$ and prednisone alone $(27 \%)$. The response rates of either CSA or CYC were significantly better than prednisone monotherapy. However, relapse of nephrotic syndrome was significantly more common after discontinuation of treatment with CSA than after IV pulse CYC. Adverse effects during the 12-month period included insulin-requiring diabetes (one with prednisone and two with CSA), pneumonia (one with prednisone and two with CSA), and localized herpes zoster (two with IVCY).

A recent pooled analysis of 65 patients with pure MLN recruited for two randomized controlled trials and who had completed 24 weeks of treatment ${ }^{31,48}$ showed no differences in the measured end points, response rate, mortality, and withdrawal rate between MMF and IV pulse CYC. ${ }^{49}$ There was also no difference in the change in proteinuria or partial response rate between MMF and CYC in those patients presenting with nephritic syndrome.

In summary, more serious MLN should be treated with a combination of glucocorticoid and a non-glucocorticoid immunosuppressive agent. A number of uncontrolled open series have reported efficacy of various regimens for MLN, such as AZA, tacrolimus, and MMF in combination with glucocorticoids. ${ }^{46}$ Many specialists start with MMF or AZA for MLN because of their lower incidence of adverse effects, reserving other agents such as IV pulse CYC, CSA, and tacrolimus for salvage therapy when the clinical response is not optimal. Newer agents, such as rituximab, infliximab, and sirolimus, should be further studied in MLN. ${ }^{37}$

\section{Conclusion}

Renal involvement is a major determinant of the prognosis of SLE. Treatment of lupus nephritis should target disease remission, prevention of relapse and complications, and long-term preservation of renal function. MMF combined with prednisone has emerged as the first-line treatment. CYC is reserved for more serious or refractory cases of lupus nephritis. The evidence for calcineurin inhibitors in lupus nephritis is less strong and they are reserved for patients intolerant or recalcitrant to MMF. While further evidence from controlled trials is eagerly awaited, the current use of B cell modulating agents is confined to recalcitrant renal disease. Novel biomarkers are being explored for earlier detection of renal flares and better prognostic stratification so that intervention can be instituted early to minimize damage to renal function. 


\section{Disclosure}

The author reports no conflicts of interest in this work.

\section{References}

1. Mok CC, Lau CS. Pathogenesis of systemic lupus erythematosus. $J$ Clin Pathol. 2003;56:481-490.

2. Oliver JE, Silman AJ. Why are women predisposed to autoimmune rheumatic diseases? Arthritis Res Ther. 2009;11:252.

3. Mok CC, Wong WS, Lau CS. Lupus nephritis in Southern Chinese patients: clinicopathologic findings and long-term outcome. $\mathrm{Am} \mathrm{J}$ Kidney Dis. 1999;34:315-323.

4. Cross J, Jayne D. Diagnosis and treatment of kidney disease. Best Pract Res Clin Rheumatol. 2005;19:785-798.

5. Mok CC. Biomarkers for lupus nephritis: a critical appraisal. J Biomed Biotechnol. Epub April 19, 2010.

6. Mok CC, Tang SS, To CH, Petri M. Incidence and risk factors of thromboembolism in systemic lupus erythematosus: a comparison of three ethnic groups. Arthritis Rheum. 2005;52:2774-2782.

7. Dooley MA, Hogan S, Jennette C, Falk R. Cyclophosphamide therapy for lupus nephritis: poor renal survival in black Americans. Glomerular Disease Collaborative Network. Kidney Int. 1997;51: $1188-1195$

8. Bastian HM, Roseman JM, McGwin G Jr, et al; LUMINA Study Group. LUpus in MInority populations: NAture vs nurture. Systemic lupus erythematosus in three ethnic groups. XII. Risk factors for lupus nephritis after diagnosis. Lupus. 2002;11:152-160.

9. Mok CC, Tang SK. Incidence and predictors of renal disease in Chinese patients with systemic lupus erythematosus. Am J Med. 2004;117: 791-795.

10. Christopher-Stine L, Siedner M, Lin J, et al. Renal biopsy in lupus patients with low levels of proteinuria. J Rheumatol. 2007;34:332-335.

11. Donadio JV Jr, Hart GM, Bergstralh EJ, Holley KE. Prognostic determinants in lupus nephritis: a long-term clinicopathologic study. Lupus. 1995;4:109-115.

12. Bono L, Cameron JS, Hicks JA. The very long-term prognosis and complications of lupus nephritis and its treatment. QJM. 1999;92:211-218.

13. Neumann K, Wallace DJ, Azen C, et al. Lupus in the 1980s: III. Influence of clinical variables, biopsy, and treatment on the outcome in 150 patients with lupus nephritis seen at a single center. Semin Arthritis Rheum. 1995;25:47-55.

14. Bakir AA, Levy PS, Dunea G. The prognosis of lupus nephritis in African-Americans: a retrospective analysis. Am J Kidney Dis. 1994;24: 159-171.

15. Nossent HC, Koldingsnes W. Long-term efficacy of azathioprine treatment for proliferative lupus nephritis. Rheumatology (Oxford). 2000;39:969-974.

16. Korbet SM, Lewis EJ, Schwartz MM, Reichlin M, Evans J, Rohde RD. Factors predictive of outcome in severe lupus nephritis. Lupus Nephritis Collaborative Study Group. Am J Kidney Dis. 2000;35:904-914.

17. Mok CC. Prognostic factors in lupus nephritis. Lupus. 2005;14: $39-44$.

18. Mok CC, Ying KY, Ng WL, et al. Long-term outcome of diffuse proliferative lupus glomerulonephritis treated with cyclophosphamide. Am J Med. 2006;119(4):355. e25-e33.

19. Mok CC, Wong RW, Lai KN. Treatment of severe proliferative lupus nephritis: the current state. Ann Rheum Dis. 2003;62:799-804.

20. Mok CC, Birmingham DJ, Leung HW, Hebert LA, Song H, Rovin BH. Vitamin D levels in Chinese patients with systemic lupus erythematosus: relationship with disease activity, vascular risk factors and atherosclerosis. Rheumatology (Oxford). 2012;51:644-652.

21. Weening JJ, D’Agati VD, Schwartz MM, Seshan SV, Alpers CE, Appel GB, et al. The classification of glomerulonephritis in systemic lupus erythematosus revisited. J Am Soc Nephrol. 2004;15:241-250.
22. Mok CC, Cheung TT, Lo WH. Minimal mesangial lupus nephritis: a systematic review. Scand J Rheumatol. 2010;39:181-189.

23. Austin HA III, Klippel JH, Balow JE, et al. Therapy of lupus nephritis: controlled trial of prednisone and cytotoxic drugs. New Engl J Med. 1986;314:614-619.

24. Gourley MF, Austin HA 3rd, Scott D, et al. Methylprednisolone and cyclophosphamide, alone or in combination, in patients with lupus nephritis. A randomized, controlled trial. Ann Intern Med. 1996;125: $549-557$.

25. Illei GG, Austin HA, Crane M, et al. Combination therapy with pulse cyclophosphamide plus pulse methylprednisolone improves long-term renal outcome without adding toxicity in patients with lupus nephritis. Ann Intern Med. 2001;135:248-257.

26. Mok CC, Lau CS, Wong RWS. Risk factors for ovarian failure in patients with systemic lupus erythematosus receiving cyclophosphamide therapy. Arthritis Rheum. 1998;41:831-837.

27. Mok CC, Ho CT, Siu YP, et al. Treatment of diffuse proliferative lupus glomerulonephritis: a comparison of two cyclophosphamide-containing regimens. Am J Kidney Dis. 2001;38:256-264.

28. Mok CC, Ho CTK, Chan KW, et al. Outcome and prognostic indicators of diffuse proliferative lupus glomerulonephritis treated with sequential oral cyclophosphamide and azathioprine. Arthritis Rheum. 2002;46: 1003-1013.

29. Houssiau FA, Vasconcelos C, D'Cruz D, et al. The 10-year follow-up data of the Euro-Lupus Nephritis Trial comparing low-dose and high-dose intravenous cyclophosphamide. Ann Rheum Dis. 2010;69:61-64.

30. Tang Z, Wang Z, Zhang HT, et al. Clinical features and renal outcome in lupus patients with diffuse crescentic glomerulonephritis. Rheumatol Int. 2009;30:45-49.

31. Appel GB, Contreras G, Dooley MA, et al. Aspreva Lupus Management Study Group. Mycophenolate mofetil versus cyclophosphamide for induction treatment of lupus nephritis. J Am Soc Nephrol. 2009;20: $1103-1112$

32. Grootscholten C, Ligtenberg G, Hagen EC, et al. Dutch Working Party on Systemic Lupus Erythematosus. Azathioprine/methylprednisolone versus cyclophosphamide in proliferative lupus nephritis. A randomized controlled trial. Kidney Int. 2006;70:732-742.

33. Bao H, Liu ZH, Xie HL, Hu WX, Zhang HT, Li LS. Successful treatment of class $\mathrm{V}+\mathrm{IV}$ lupus nephritis with multitarget therapy. $J \mathrm{Am}$ Soc Nephrol. 2008;19:2001-2010.

34. Chen W, Tang X, Liu Q, et al. Short-term outcomes of induction therapy with tacrolimus versus cyclophosphamide for active lupus nephritis: a multicenter randomized clinical trial. Am J Kidney Dis. 2011;57: 235-244.

35. Mok CC, Ying KY, Tong KH, et al. Mycophenolate mofetil versus tacrolimus for active lupus nephritis: an extended observation of a randomized controlled trial. Arthritis Rheum. 2008;58(Suppl 9):S566.

36. Rovin BH, Furie R, Latinis K, et al. LUNAR Investigator Group. Efficacy and safety of rituximab in patients with active proliferative lupus nephritis: the lupus nephritis assessment with rituximab (LUNAR) study. Arthritis Rheum. 2012. In press.

37. Jónsdóttir T, Gunnarsson I, Mourão AF, Lu TY, van Vollenhoven RF, Isenberg D. Clinical improvements in proliferative vs membranous lupus nephritis following B-cell depletion: pooled data from two cohorts. Rheumatology (Oxford). 2010;49:1502-1504.

38. Melander C, Sallée M, Trolliet P, et al. Rituximab in severe lupus nephritis: early B-cell depletion affects long-term renal outcome. Clin J Am Soc Nephrol. 2009;4:579-587.

39. Vigna-Perez M, Hernández-Castro B, Paredes-Saharopulos O, et al. Clinical and immunological effects of Rituximab in patients with lupus nephritis refractory to conventional therapy: a pilot study. Arthritis Res Ther. 2006;8:R83.

40. Illei GG, Takada K, Parkin D, et al. Renal flares are common in patients with severe proliferative lupus nephritis treated with pulse immunosuppressive therapy: long-term follow-up of a cohort of 145 patients participating in randomized controlled studies. Arthritis Rheum. 2002;46:995-1002. 
41. Moroni G, Doria A, Mosca M, et al. A randomized pilot trial comparing cyclosporine and azathioprine for maintenance therapy in diffuse lupus nephritis over four years. Clin J Am Soc Nephrol. 2006;1:925-932.

42. Contreras G, Pardo V, Leclercq B, et al. Sequential therapies for proliferative lupus nephritis. $N$ Engl J Med. 2004;350:971-980.

43. Moroni G, Gallelli B, Quaglini S, et al. Withdrawal of therapy in patients with proliferative lupus nephritis: long-term follow-up. Nephrol Dial Transplant. 2006;21:1541-1548.

44. Houssiau FA, D'Cruz D, Sangle S, et al. MAINTAIN nephritis trial group. Azathioprine versus mycophenolate mofetil for long-term immunosuppression in lupus nephritis: results from the MAINTAIN Nephritis Trial. Ann Rheum Dis. 2010;69:2083-2089.

45. Dooley MA, Jayne D, Ginzler EM, et al. Mycophenolate versus azathioprine as maintenance therapy for lupus nephritis. $N$ Engl J Med. 2011;365:1886-1895.
46. Mok CC. Membranous nephropathy in systemic lupus erythematosus: a therapeutic enigma. Nat Rev Nephrol. 2009;5:212-220.

47. Austin HA 3rd, Illei GG, Braun MJ, Balow JE. Randomized, controlled trial of prednisone, cyclophosphamide, and cyclosporine in lupus membranous nephropathy. J Am Soc Nephrol. 2009;20:901-911.

48. Ginzler EM, Dooley MA, Aranow C, et al. Mycophenolate mofetil or intravenous cyclophosphamide for lupus nephritis. $N$ Engl J Med. 2005;353:2219-2228.

49. Radhakrishnan J, Moutzouris DA, Ginzler EM, Solomons N, Siempos II, Appel GB. Mycophenolate mofetil and intravenous cyclophosphamide are similar as induction therapy for class $\mathrm{V}$ lupus nephritis. Kidney Int. 2010;77:152-160.

International Journal of Women's Health

Dovepress

\section{Publish your work in this journal}

The International Journal of Women's Health is an international, peerreviewed open-access journal publishing original research, reports, reviews and commentaries on all aspects of women's healthcare including gynecology, obstetrics, and breast cancer. Subject areas include: Chronic conditions (migraine headaches, arthritis, osteoporosis);
Endocrine and autoimmune syndromes; Sexual and reproductive health; Psychological and psychosocial conditions. The manuscript management system is completely online and includes a very quick and fair peer-review system. Visit http://www.dovepress.com/ testimonials.php to read real quotes from published authors. 